\title{
One Stop Crisis Management Centers: A Commendable Intervention to Stop Violence in the Lives of Children and Families
}

\author{
Rekha L Rana
}

\begin{abstract}
This brief report captures the work of OCMCs established by the Ministry of Health in Nepal to alleviate gender based violence since 2011 .
\end{abstract}

\section{Keywords-GBV, OCMCs, Ministry of Health,}

\section{Background}

Over the last four years (2011 onwards), the Ministry of Health and Population (MoHP) has established seventeen OCMCs in each of sixteen districts (Dang, Doti, Panchthar, Solukhumbu, Sunsari, Kathmandu, Kavre, Jumla, Saptari, Bardiya, Tanahu, Hetauda, Baglung, Sarlahi, Kanchanpur and Nawalparasia) cross Nepal with technical backstopping from Nepal Health Sector Support Program (NHSSP)/Options with the fund support from DFID. Each OCMC aims to provide an integrated package of services for survivors of violence through a 'one-door' system that follows three core principles: (i) ensuring the security and safety of GBV survivors; (ii) maintaining confidentiality, and (iii) respecting the dignity, rights and wishes of survivors at all times.

OCMCs are designed to follow a multi-sectoral and locally coordinated approach to provide GBV survivors with a comprehensive range of services including health care, psycho-social counselling, access to safe homes, legal protection, personal security and vocational skills training.

\section{Findings ${ }^{1}$}

Overall, the district report showed that from October 2013 - October 2015, OCMCs have provided essential services required by violence survivors. From the data available, a total of 2,273 individuals - 2173 (96\%) women and $100(4 \%)$ men - have benefitted from services since the establishment of OCMCs. Within this total 2833, 1,504 data from other districts showed 176 cases in Baglung, 167 in Kanchanpur, 128 in Makwanpur and 110 in Doti. Aside from physical assault/domestic violence cases

\section{Rekha Rana}

GESI Coordinator

NHSSP/Options, MoHP, Kathmandu Nepal

\footnotetext{
${ }^{1}$ The findings are based on the presentations made by the district representatives. Some districts lacked information on the types of services provided, referrals and GBV age categories. Accordingly, these data are not representative of all 16 OCMCs. However, with regard to numbers and the types of violence, it seems that survivors facing multiple forms of violence (e.g. domestic violence and mental abuse) have been counted for both types of violence, hence the total number of beneficiaries is higher than expected in the table 1.
}

sexual violence (mostly rape) was the most common and accounted for $26 \%$ of all cases. Kathmandu (Maternity Hospital) reported that among its 356 GBV cases reported, 295 related to sexual violence, mostly rape. Rape was also prominent in other districts as follows: Sunsari (67 cases), Makwanpur (60), Bardiya (55) and Baglung (52).

A total of $455(16 \%)$ cases of mental torture were reported with Dang (168), Kanchapur (130) having the highest counts. Data from the other districts are also cause for concern.

In all districts, 907 (43.5\%) of cases were self-referrals (including via other hospital units), 783 (37.5\%) from the Police, $212(10.1 \%)$ from local agencies (including safe homes), 95 (4.5\%) from NGOs and 90 (4.3\%) from other sources $^{2}$. Earlier data showed that fewer than a dozen cases were self-referrals and this suggests that levels of public awareness of OCMC service availability may have increased. District staff also reported that women are becoming more vocal and assertive in seeking OCMC support services. In addition, while much remains to be done to improve local inter-agency coordination and collaboration, basic cross-sectoral functionality was reported.

Age related GBV data showed that women in the 15-49 years cohort were most likely to be affected. A total of 2,096 (78.5\%) women were GBV victims followed by 312 (14.9\%) from the 0-14 age group, $110(5.3 \%)$ from the 5065 group and $29(1.4 \%)$ from 65 years and above. This suggests that married women and/or those with a family or partner suffer most. The high number of physical assault/domestic violence cases reported correlates with this data.

Regarding OCMC service utilization, medical treatment - ranging from basic medical check-ups and psycho-social counselling, to medico-legal services - were the most common.

\section{Good Practices by OCMCs}

- Coordination and referral of GBV cases from other units, departments and emergency sections of hospitals to the OCMC.

- 24 hours services for OCMC cases at hospitals.

- Information dissemination about OCMC through various media, such as FM radio, brochures/pamphlets.

- Cases referred from the hospitals being taken seriously by the Police, safe homes, WDO, rehabilitation centres and attorneys.

\footnotetext{
${ }^{2}$ Neighbours, friends/relatives, Child Centres.
} 
Proc. of the Third Intl. Conf. on Advances in Management, Economics and Social Science - MES 2015.

Copyright (C) Institute of Research Engineers and Doctors, USA .All rights reserved.

ISBN: 978-1-63248-081-1 doi: 10.15224/ 978-1-63248-081-1-79

- At a local level, orientation on OCMC services being provided by WDO, DPHO, and DEO to their staff.

- Coordination and support from WDO, the Police and others for the cases requiring long-term rehabilitation.

- Support from UNFPA, WOREC for effective functioning and management of OCMC.

\section{Recommendations}

- Integration into the hospital system: treating OCMC as one of the units of the hospital and not a separate entity.

- Strong coordination, communication and collaboration among the stakeholders is crucial.

- Information about OCMC should be widely disseminated via media and other methods.

- Multi-year contracts for OCMC staff nurses would help retain staff.

- The maximum number of health staff, as well some leaders of the community, must be well-informed and well-trained about the centre, its function as well as its importance.

- GBV sensitization training for health staff of OPD, indoor and emergency, and laboratory staff of the hospital where the centre is established.

- Orientation for all members of the district coordination committee.

- Timely release of budgets.

- Develop a referral and clinical protocol, and a separate protocol on rape and cases with serious injuries or psychological trauma which may need treatment at a more specialised centre or require higher level legal advice.

- Availability of a post rape kit.

- Regular supervision and monitoring from central and regional levels is a must.

- Development of umbrella guidelines for effective coordination and collaboration among the stakeholders is key.

\section{v. V. Conclusion}

Experiences from across the world indicate that managing violence, especially gender based violence (GBV) is a very sensitive issue, demanding extensive technical know-how, commitment, patience and coordination to address the needs of survivors, their family members and the perpetrators. OCMCs are a new and challenging initiative. But the challenges can be overcome through improved awareness raising activities; capacity building of OCMC staff and stakeholders; survivor follow-up; improved screening and coordinating strategies; and more social protection activities to support survivors. The implementation of these measures will demand consistent and coordinated monitoring from ministries and institutions to district line agencies. This will also require that other government agencies, beyond MoHP alone, work more systematically to prevent and address GBV in a holistic manner. OCMCs should also coordinate and collaborate with district health offices, women and children's offices and district police offices in order to raise awareness, advocate against violence against women and lobby for support to survivors. Supporting the establishment of OCMCs in all 75 districts of the country to support survivors affected by various forms of violence is the need of the hour. 